\title{
The Most Common Taekwondo Kicks using the eShield at the 23rd Asian Taekwondo Championships, Vietnam 2018
}

\author{
Abed Aljawad Bashar, Assistant Professor \\ An-najah Notional University \\ Abdul Fattah Osama, PhD \\ The Ministry of Education, Jordan
}

Doi: 10.19044/esj.2018.v14n27p346 URL:http://dx.doi.org/10.19044/esj.2018.v14n27p346

\begin{abstract}
This paper focuses on identifying the most common taekwondo hits and kicks used by players at the 23rd Asian taekwondo championships, Vietnam 2018. A lot of researchers attended the 23rd Asian taekwondo championships and they analyzed the quarterfinals, semifinals, and finals for all weights classes for both males and female players. 111 matches (55 for male and 56 for females) were analyzed in this study. A form was used to process and analyze the matches. The results of this study showed that the turn kick attack with the rear leg at the abdominal level was the most commonly used kick, with the highest rate among male and female players $(41.4 \%$ males and $46.3 \%$ females). Also, the percentage of the offensive kicks and strategies among males and females was very high compared with the defensive kicks and strategies. In this regard, the percentage of the offensive kicks among the males was $91 \%$, whereas the rate of the defensive kicks reached $9 \%$. On the other hand, the rate of the offensive kicks among the females was 88\%, whereas the rate of the defensive kicks reached $12 \%$. It was concluded that the offensive kicks at the abdominal level was the most commonly used kick among the players. In addition, there was a significant increase in the rate of the face kicks after the regulations amendments.
\end{abstract}

Keywords: Gender, Training, Coaching, Taekwondo, Kicks

\section{Introduction}

Modern sport of Taekwondo originated in South Korea after World War II, after the Korean troops returned from Japan (Romin, et al., 2009). Thereafter, the Taekwondo turned into game sport that gained international exposure and widespread publicity. It became one of the most important martial art games, particularly after it had been included in the Olympic Games 
in Sydney 2000. It was adopted as an official game by the International Olympic Committee. This led to a remarkable increase in its popularity among countries and players, leading to a massive number of fans and practitioners around the world. The World Taekwondo Federation (WTF) statistics show that there are more than 40 million players in more than 160 countries (Mohsen, et al., 2010). However, there is no doubt that the best achievement Taekwondo had is that it became the main target of most competent players, coaches, and even states.

The system of competitions or contests in the Taekwondo is not much different from the other self-defense sports. Each player tries to win by scoring the highest number of points. The player uses his hand to hit the abdomen or chest or uses his leg to kick the body or the head of his opponent. Usually, the player's kicks are aimed at the opponent's shielded chest, abdomen, head or face. The scoring punches and kicks are counted by the referees.

Due to increased interest in Taekwondo, a Protection Scoring System was introduced to safeguard the player's rights without favoritism or discrimination. The new Taekwondo regulations are aimed at the prevention of judgment errors; it is not quite an easy task for the referees to observe the players' kicks due to the fast sequence of the kicks. Therefore, the World Taekwondo Federation has set judgment rules, regulations, and codes for the Olympic Taekwondo. To record the best results, players now wear sensors chest shields as well as head eShields that work automatically and score points when they are kicked. Therefore, this protective gear led to a significant decrease in the judgment errors and gave the players greater opportunities to score points.

The Taekwondo sensors on the abdomen, chest or head are used to indicate impact, and are used for scoring in Taekwondo. They send signals to the linked computer by Bluetooth to score points for the competitor who kicks it. This happens when the foot of the competitor who wears the sensor socks touches the sensor handguard; when both sensors come into contact with each other, a point is scored. The sensors in more than one place in the competitor's socks compel him to use various kicks with the front or rear parts of the foot. This gear gave the competitors the ability to vary their kicks and bunches. Nevertheless, they were limited to certain kicks only in the past.

The protective equipment gave the competitors the opportunity to use the highest number of the scoring kicks due to the sensor socks. The World Taekwondo Federation's judgment rules and regulations made the game even more exciting than ever. The scoring system distinguishes between kicks on the abdomen and chest and the kicks on the head. One point is usually scored for the abdominal and chest kicks, and three points for kicks on the head. Besides, an extra point is scored for both abdominal and head kicks if the kick 
is cyclical (WTF, 2007). Also, a point can be scored by a hand punch. This scoring diversity leads to various types of scoring kicks.

The researchers, however, noticed that the increase in the number of the competitors' use of the head kicks is due to the new scoring system that provides better chances for scoring higher points. The study problem aims to highlight the most important and most frequently scoring kicks in the 2014 Asian games, Incheon, South Korea. Furthermore, the study aims to provide the players and coaches with an opportunity to focus on these scoring kicks, which would enhance the level of scoring and thus increase the competitors' winning chances.

Consequently, the researchers believe that analysis of Taekwondo matches in international competitions plays an important role in modern training and future decision-making strategies, which thus enhance performance and achievement. The present study examined the most common movements in the Taekwondo matches; highlight the common and less common scoring movements; and assists coaches in planning training that is focused more on the most used movement.

\section{Materials and Methods}

The study consisted of 111 matches in the 23rd Asian taekwondo championships, Vietnam 2018. For both gender, we analyzed the quarterfinals, semifinals, and finals of all weights level by both male and female players. The researchers designed a form to record the information for each game and the data collected was entered for computer analyzation.

\section{Research Variables:}

The study variables included the following Taekwondo kicks:

- Slip turn kick attack with the rear leg - abdomen.

- Slip turn kick attack with the rear leg - face.

- Turn kick withdrawal with the front leg - abdomen.

- Turn kick withdrawal with the front leg - face.

- Slip turn kick attack with the front leg - abdomen.

- Slip turn kick attack with the front leg - face.

- $\quad$ Rise kick attack with the front leg - face.

- Rise kick attack with the rear leg - face.

- Push kick attack with the front leg - abdomen.

- Push kick attack with the rear leg - abdomen.

- Screw turn attack with the front leg - abdomen.

- Screw turn attack - face.

- Backside attack on the abdomen.

- Backside attack on the face.

- Backside withdrawal on the abdomen. 
- Backside withdrawal on the face.

- Back turn attack on the face.

- Back turn withdrawal on the face.

- Hook kick with the front leg on the face.

- Hook kick with the rear leg on the face.

\section{Statistical Analyses}

Statistical analysis was carried out using SPSS package (version 10). Both frequencies and percentages were used in analyzing the data. Additionally, a value of $\mathrm{p}<0.05$ was considered to be statistically significant.

\section{Results}

Based on observation and analysis, Table 1 shows the occurrences and the percentages of taekwondo kicks used by every player during the games.

Table 1. Occurrences and percentages of players' kicks numbers

\begin{tabular}{|l|l|l|}
\hline Taekwondo kicks & Occurrences & Percentages \\
\hline Turn kick attack with rear leg - abdomen & 201 & $41 \%$ \\
\hline Turn kick attack with rear leg - face & 8 & $1.6 \%$ \\
\hline Turn kick withdrawal with front leg - abdomen & 12 & $2.5 \%$ \\
\hline Turn kick withdrawal with front leg - face & 5 & $1.02 \%$ \\
\hline Slip turn kick attack with front leg - abdomen & 98 & $20.7 \%$ \\
\hline Slip turn kick attack with front leg - face & 46 & $9.5 \%$ \\
\hline Rise kick attack with front leg - face & 9 & $1.9 \%$ \\
\hline Rise kick attack with rear leg - face & 10 & $2.1 \%$ \\
\hline Push kick attack with front leg - abdomen & 33 & $6.8 \%$ \\
\hline Push kick attack with rear leg - abdomen & 32 & $6.6 \%$ \\
\hline Screw turn click attack - abdomen & 0 & $0 \%$ \\
\hline Screw turn click attack - face & 0 & $0 \%$ \\
\hline Back side attack on the abdomen & 1 & $0.21 \%$ \\
\hline Back side attack on the face & 0 & $0 \%$ \\
\hline Back turn withdrawal on the abdomen & 3 & $0.62 \%$ \\
\hline Back turn withdrawal on the face & 0 & $0 \%$ \\
\hline Back turn attack on the face & 2 & $0.41 \%$ \\
\hline Back turn withdrawal on the face & 0 & $0 \%$ \\
\hline Hook click with front leg on the face & 1 & $0.21 \%$ \\
\hline Hook click with rear leg on the face & 0 & $0 \%$ \\
\hline Punch hand on chest protector & 25 & $5.1 \%$ \\
\hline Total & 486 & $100 \%$ \\
\hline
\end{tabular}

Table 1 shows the numbers and percentages of kicks performed by the players. The results of this study show that the kicks that achieved the highest frequencies and percentages are as follows respectively: turn kick attack with the rear leg at the abdominal level, slip turn kick attack with front leg at the abdominal level, slip turn kick attack with the front leg on the face level, push kick attack with the rear leg at the abdominal level, hand punch on the 
abdominal level. The percentages are as follows respectively: $41.4 \%, 20.7 \%$, $9.5 \%, 6.8 \%, 6.6 \%$, and $5.1 \%$. The study results also revealed that the kicks that achieved the lowest frequencies and percentages are as follows respectively: hook kick with the front leg on the face level, backside kick attack on the abdominal level, back turn kick at the face level, backside kick withdrawal at the abdominal level, turn kick withdrawal with the front leg at the face level, turn kick attack with the rear leg at the face level. The percentages are as follows respectively: $0.21 \%, 0.21 \%, 0.42 \%$, and $0.62 \%$, $1.02 \%, 1.6 \%$.

Also, Table 1 shows that some kicks were not used in all the matches that were observed, including: screw turn kick attack at the abdominal level, screw turn kick attack at the face level, backside kick attack at the face level, backside kick withdrawal at the face level, turn kick withdrawal with the front leg at the face level, turn kick attack with the rear leg at the face level. The table also shows that the percentage of the total number of the offensive kicks and strategies is $91 \%$, while the percentage of the total number of the defensive kicks and strategies is $9 \%$.

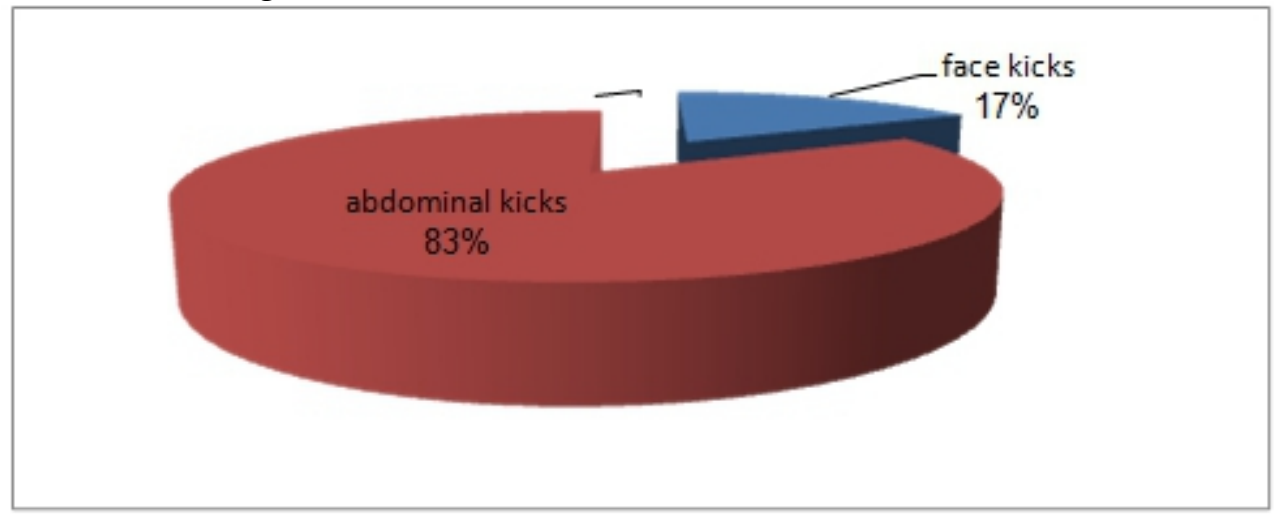

Figure 1. Kicks percentages at the levels of the face and abdomen

In addition, Figure 1 shows the percentage of the kicks at the levels of the face and abdomen in all the matches. The percentage number of the abdominal kicks is $83 \%$, whereas the percentage number of the kicks at the face level is $17 \%$.

Figure 2 shows the percentage of the kicks using the front leg and rear leg in the observed matches. The percentage of the number of kicks using the front leg is $43 \%$, while the percentage of the number of kicks using the rear leg is $53 \%$. 


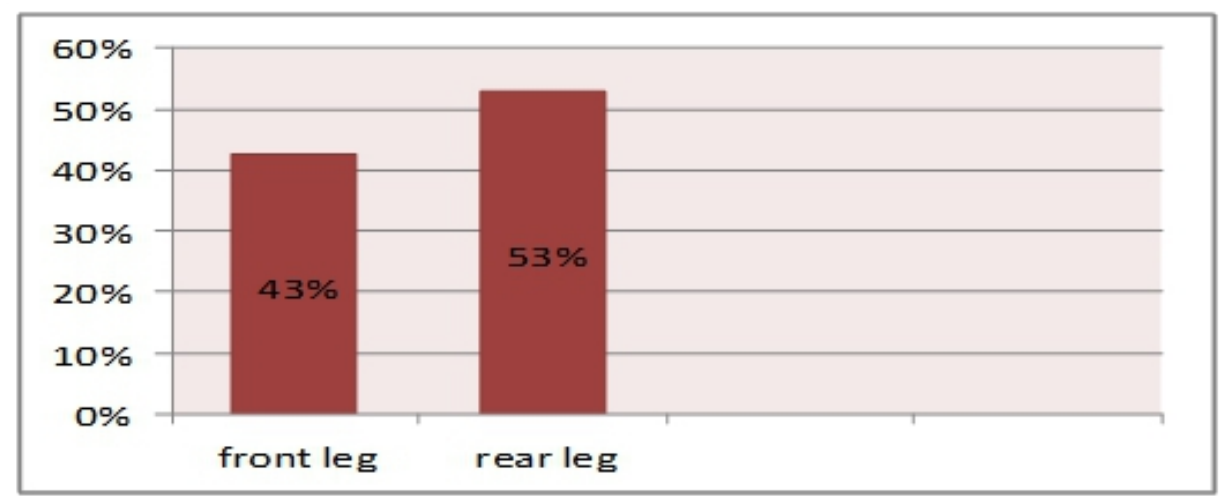

Figure 2. Kicks percentages using the players' front and rear legs

Furthermore, Table 2 shows kick numbers used by the players in the observed matches.

Table 2. Occurrences and percentages of the number of the players' kicks

\begin{tabular}{|l|l|l|}
\hline Taekwondo kicks & Occurrences & Percentages \\
\hline Turn kick attack with rear leg - abdomen & 144 & $46.3 \%$ \\
\hline Turn kick attack with rear leg - face & 14 & $4.5 \%$ \\
\hline Turn kick withdrawal with front leg - abdomen & 14 & $4.5 \%$ \\
\hline Turn kick withdrawal with front leg - face & 10 & $3.2 \%$ \\
\hline Slip turn kick attack with front leg - abdomen & 27 & $8.7 \%$ \\
\hline Slip turn kick attack with front leg - face & 49 & $15.8 \%$ \\
\hline Rise kick attack with front leg - face & 10 & $3.2 \%$ \\
\hline Rise kick attack with rear leg - face & 11 & $3.5 \%$ \\
\hline Push kick attack with front leg - abdomen & 11 & $3.5 \%$ \\
\hline Push kick attack with rear leg - abdomen & 3 & $1.00 \%$ \\
\hline Screw turn click attack - abdomen & 0 & $0 \%$ \\
\hline Screw turn click attack - face & 0 & $0 \%$ \\
\hline Back side attack on the abdomen & 3 & $1.00 \%$ \\
\hline Back side attack on the face & 0 & $0 \%$ \\
\hline Back side withdrawal on the abdomen & 4 & $1.3 \%$ \\
\hline Back side withdrawal on the face & 1 & $0.32 \%$ \\
\hline Back turn attack on the face & 2 & $0.64 \%$ \\
\hline Back turn withdrawal on the face & 1 & $0.32 \%$ \\
\hline Hook click with front leg on the face & 0 & $0 \%$ \\
\hline Hook click with rear leg on the face & 0 & $0 \%$ \\
\hline Hand punch on chest protector & 7 & $2.3 \%$ \\
\hline Total & 311 & 100 \\
\hline
\end{tabular}

Table 2 shows the number and percentages of kicks performed by the players. kicks achieved the highest frequencies and percentages as follows respectively: turn kick attack by rear leg on the abdomen, slip turn kick attack with front leg on the face, slip turn kick attack with front leg on the abdomen, turn kick attack with rear leg on the face, turn kick withdrawal with front leg on the abdomen, push kick with front leg on abdomen, rise kick with rear leg 
at face level. The percentages are as follows respectively: $46.3 \%, 15.8 \%$, $8.7 \%, 4.5 \%, 4.5 \%, 3.5 \%$, and $3.5 \%$. The results also show that the kicks achieved the lowest frequencies and percentages as follows respectively: back turn kick withdrawal at the face level, backside kick withdrawal at the face level, back turn kick attack at the face level, backside kick attack at the abdominal level, push kick attack with rear leg at the face level, backside kick withdrawal at the abdominal level. The percentages are as follows respectively: $(0.32 \%, 0.32 \%, 0.64 \%$, and $0.62 \% 1 \%, 1 \%, 1.3 \%$.

The results of this study show that some kicks were not used in all the matches that were observed including: screw turn kick attack at the abdominal level, screw turn kick attack at the face level, backside kick attack at the face level, hook kick with rear leg at the face level, hook kick with rear leg at the face level. The table also shows that the percentage of the total number of the offensive kicks and strategies is $88 \%$, while the percentage of the total number of the defensive kicks and strategies is $12 \%$. The percentage of the kicks at the levels of the face and abdomen in all the matches is given in Figure 3 below. Thus, Figure 3 shows the abdominal percentage kicks is 69\%, whereas kicks at the face level percentage is $31 \%$.

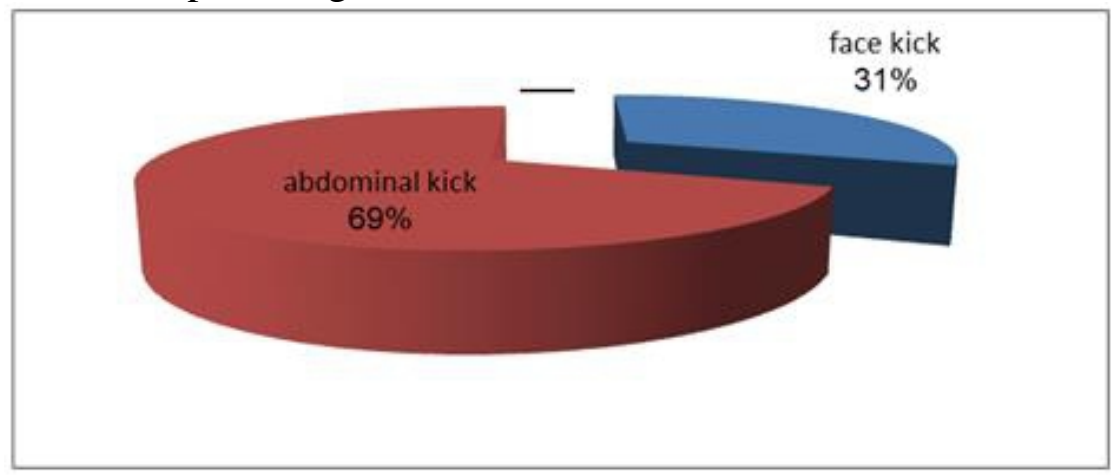

Figure 3. Kicks percentages using the players' front and rear legs

However, Figure 4 shows the kicks percentage number using front and rear legs in all the matches. Kicks using the front leg percentage are 39\%, whereas kicks using the rear leg percentage are 59\%. 


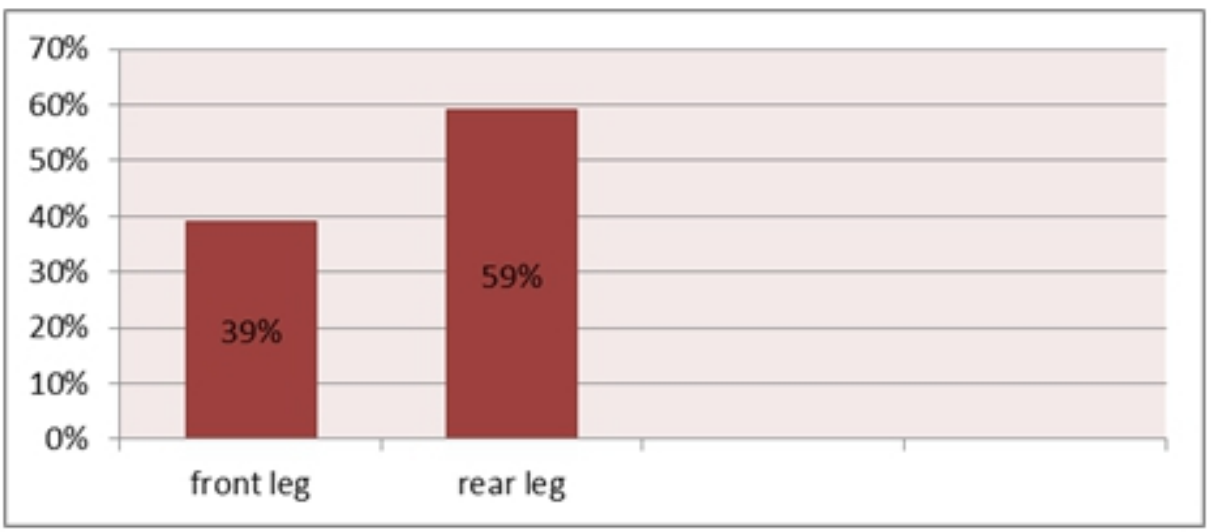

Figure 4. Percentages of the kicks using the players' front and rear legs

\section{Discussion}

Turn kick attack with rear leg at the abdominal level achieved the highest rate of occurrence among males and female players (males: $41.4 \%$, females: $46.3 \%$ ). The occurrence of this kick by the players show the importance of both speed and power in the game of Taekwondo (Marcovic et al., 2008; Butios \& Tasika, 2007). It gives the players the ability to follow up and repeat kicks at the opponent, without any spinning or difficult movements, and thus its rate reached $72.7 \%$ (Tze luk, et al., 2001). They argue that turn kick attack with rear leg at the abdominal level momentum is higher than any other types of kicks. Indeed, the momentum is one of the most important things since it helps the players to resist and fight the opponent. In addition, a player can attack and kick so hastily that he is more likely to score higher points.

Moreover, the study show that slip turn attack at the abdominal level and slip turn attack at the face level came in the second and third positions with the rates of $20.7 \%$ and $9.5 \%$ respectively. As for female players, slip turn attack at the face level, and slip turn attack at the abdominal level, came in second and third positions with the rates of $15.8 \%$ and $8.7 \%$ respectively. This is because the front leg is nearer to the opponent than the rear leg; however, it is less powerful than kick with the rear leg. Luk and Hong (2000) argued that the time needed to kick with the front leg is shorter than the time needed for kicking with the rear leg. Obviously, the speed of the kicks is one of the most important things that players need in order to avoid the opponent's kicks and attacks.

The researchers found out that there was an increase in the use of the push click with front leg at the abdominal level by both male and female players. It came in the fourth position among the males with a percentage of $6.5 \%$. As for females, it was in the fifth position with a percentage of $3.5 \%$. These rates are good compared with the percentage of $2.02 \%$ according to the study of Tze, Luk, et al. (2001). In the recent years, there has been an increase 
in the use of such powerful kick that it became an essential part of modern Taekwondo. The new electronic scoring system played a vital role in this; the sensor socks allow the player to score a point when his foot touches the eShield. In addition, the kick has become a defensive technique since it gives the player the opportunity to stop the opponent's attacks, and since the bend of the knee deters the opponent from reaching the body shield.

The study also shows that some kicks have not been used. These kicks include screw turn kick attack at the face and abdominal levels, backside kick attack at the face level, backside kick withdrawal at the face level, turn kick withdrawal with the front leg at the face level, turn kick attack with the rear leg at the face level, and hook kick attack with the rear leg. First, all of them are directed at either the head or face. In this regard, it is not an easy task to focus the kick towards the head because this requires an additional effort. Therefore, players often defend their heads with their arms to avoid the knockout. Second, such kicks are of martial-art-like spinning that require highdemanding techniques. Besides, the player may lose his balance during the spinning and fall on the ground, which typically leads to penalty. Player may lose his ability to defend himself, making him prone to opponent's kicks on the face and abdominal level.

The results show that the rate of using the offensive kicks and techniques among both male and female players was very high when compared with the defensive ones. For males, the rate of the offensive kicks was $91 \%$, while the rate of the defensive ones was $9 \%$. As for females, the offensive kicks achieved a rate of $88 \%$, while the rate of the defense kicks was $12 \%$. This increased number of offensive attacks in the Taekwondo was due to regulations amendments that banned passive play for more than ten seconds. However, each player loses half a point in this case. Therefore, the players are compelled to attack each other during the whole match. At the same time, the offensive attacks are likely to force the opponent to retreat from the play area. According to The World Taekwondo Federation's amendments in 2014, a competitor is likely to lose the competition if he receives ten warnings.

There are remarkable changes in the Taekwondo techniques after the adoption of the new Taekwondo regulations. Casolino (2012) highlighted the importance of offensive kicks and techniques in his study which states that the rate of the offensive kicks reached $91.6 \%$, whereas the defensive kicks reached $8.4 \%$ only. The new scoring regulations led to a variety of kicking techniques. The rate of the offensive attacks was comparably similar to the defensive ones. According to Tze, et al. (2001) study, the rate of the offensive kicks was $55.9 \%$, while the rate of the defensive kicks was $42.8 \%$.

Figures 1 and 3 show that the rate of using the abdominal kicks is higher than the rate of the kicks at the face level (males: the abdominal rate $(83 \%)$, face rate $(17 \%)$; females: abdominal rate $(69 \%)$, face rate $(31 \%))$. The 
researcher suggests that the reason for this higher rate of the abdominal kicks is due to the high velocity of the abdominal kicks, as well as the need of the face kicks for greater muscular efforts. According to Luk and Hong (2000) and Piete and Pieter (1995), the performance of the abdominal kicks at the head level requires greater muscular strength. Therefore, many players prefer the attack kicks at the abdominal level in order to maintain a higher level of performance for the longest possible period.

Moreover, the kicks at the abdominal level give the players greater chances for sequence and speed, thus increasing their chances of scoring. Despite this increased rate of abdominal kicks, there was a prominent increase in the face kicks after the Act amendments in 2001 (WTF, 2007). The new act allowed three points for every kick at the level of the face. According to the present study, the rate of the face kicks was $17 \%$ among the male players and $31 \%$ among the female players, which is a high rate compared with the previous studies before 2007. According to Tze, et al. (2001), the rate of the abdominal kicks was $91.4 \%$ and the rate of face kicks was $8.6 \%$.

Figures 2 and 4 show that the rate of using kicks with rear legs is higher than the front legs (male: rear leg (53\%) front leg (43\%); female: rear leg $(59 \%)$, front leg (39\%)). The researcher believes that the high rate of using the rear legs is attributed to the speed and momentum of the rear legs. Therefore, the new electronic scoring system registers the point when the body shield is kicked strongly enough. Also, its power is set up according to the weights of the players.

Casolino (2012) pinpoints the high rate of kicks with the rear legs (94.4) - the rate of the front leg was only 5.6\%. According to Tze, et al. (2001), the rate of the kicks with the rear legs was $75.9 \%$, and the kicks with the front legs was $24.1 \%$. Despite the increased rate of the kicks with the rear legs, there is, however, an increase in the rate of the kicks with the front legs (male $43 \%$, female 39\%). On the other hand, Caroline's study (2012) found out that the kicks with the front legs reached 5.6\%. According to Tze, et al. (2001), the rate was $24.1 \%$. The researcher argues that the increased use of the front leg at the level of the face is attributed to the regulations amendments, which granted three-point score.

The study also revealed that there is a noticeable increase in the use of scoring hand punch, compared with previous studies (male 5.1\%, female $2.3 \%$ ). Thus, this punch has become one of the defensive techniques, especially with the push kick and the lifting of the opponent. It actually gives the player the chance to direct an effective punch at the opponent's body shield, which may lead to his imbalance and standing on one foot. 


\section{Conclusion}

In conclusion, the results of this study show that the turn kick with the rear leg at the abdominal level is the most important and most frequent Taekwondo kick among male and female competitors. The Taekwondo kicks at the abdominal level are more widely popular than the kicks at the face level among male and female competitors.

The rate of the offensive kicks is higher than the defensive kicks among male and female competitors. Despite the higher rate of the abdominal kicks, there is a significant increase in the rate of using the kicks at the face level, due to the recent amendments, which added three point scoring scheme. Furthermore, there is an increase in the rate of using hand punch at the level of the chest shield.

\section{References:}

1. Butios, S. \& Tasika, N. (2007). Changes in heart rate and blood lactate concentration as intensity parameters during simulated Taekwondo competition. J Sports Medicine and Physical Fitness; 47 (2):179-85 [PubMed].

2. International Symposium on Biomechanics in Sports (Vol. II, pp. 920924). Hong Kong: The Chinese University of Hong Kong.

3. Luk, T. \& Hong, Y. (2000). Comparison of electromyography activity between different types of Taekwondo round-house kick. In Y. Hong \& D. P. Johns (Eds.), Proceedings of XVIII.

4. Markovic, G., Vucetic, V., \& Cardinale, M. (2008). Heart rate and lactate responses to taekwondo fight in elite women performers. Biol Sport; 25(2):135-146.

5. Mohsen Kazemi, Giovanni Perri, \& David Soave (2010). A profile of 2008 Olympic Taekwondo competitors, the journal of Canadian chiropractic association, 54(4): 243-249.

6. Pieter, F. \& Pieter, W. (1995). Speed and force in selected taekwondo techniques. Biology of sport, 12(4), 257-266.

7. Ramin, Kordi, Nicola, Maffulli Randall, Wroble, Angus, \& Wallace (2009). Combat Sports Medicine. pp 263-286.

8. Tze Chung Luk, Youlian Hong \& Danny Chu, P. K. (2001). Analysis of Strategy Used in Taekwondo Competition.19 International Symposium on Biomechanics in Sports. University of San Francisco.166-169.

9. World Taekwondo Federation competition roles [Online] (2007). August 6. Cited 2008 May 15]. Available from: URL: http://www.wtf.org.

10. World Taekwondo Federation competition roles [Online] (2014). Available from: URL: http://www.wtf.org 